Recepción: 20 / 04 / 2017

Aceptación: 20 / 05 / 2017

Publicación: 15 / 06 / 2017

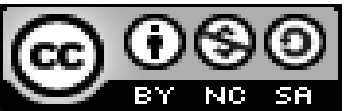

Ciencias de la Computación Artículo de Investigación

\title{
Hardening y diseño de un ambiente de virtualización para el manejo de múltiples servidores de VoIP sobre una misma plataforma
}

\section{Hardening and designing a virtualization environment for managing multiple VoIP servers on the same platform}

Endurecimento e design de um ambiente de virtualização para gerenciar vários servidores em um VoIP plataforma

Eleanor A. Varela-Tapia ${ }^{\text {I }}$

eleanor.varelat@ug.edu.ec

Diana G. Albán-Loor ${ }^{\text {II }}$ diana.albanl@ug.edu.ec

Iván L. Acosta-Guzmán III ivan.acostag@ug.edu.ec

Jenniffer M. Yagual-Lozano ${ }^{\text {IV }}$ jenniffer.yaguall@ug.edu.ec

Correspondencia: eleanor.varelat@ug.edu.ec

I. Magister en Sistemas de Información Gerencial; Magister en Docencia y Gerencia en Educación Superior; Diploma Superior en Diseño Curricular por Competencias; Ingeniera en Computación; Docente Universidad de Guayaquil, Guayaquil, Ecuador.

II. Ingeniera en Networking y Telecomunicaciones; Universidad de Guayaquil, Guayaquil, Ecuador.

III. Magister en Sistemas de Información Gerencial; Magister en Administración de Empresas; Ingeniero en Computación; Docente Universidad de Guayaquil, Guayaquil, Ecuador.

IV. Ingeniera en Networking y Telecomunicaciones; Universidad de Guayaquil, Guayaquil, Ecuador. 


\section{Resumen}

El servicio de telefonía es un medio de comunicación indispensable en las empresas que permite realizar todo tipo de actividad comercial o personal. El uso de esta tecnología radica en: la calidad de servicio que ofrecen a un costo bajo, la disponibilidad en todo momento, la accesibilidad sin interrupciones o congestionamiento y la facilidad de uso sin dejar a un lado la seguridad y escalabilidad a través del tiempo. Debido a los múltiples avances tecnológicos constantes y la innovación de tecnologías open source y virtualización, es sugerido la implementación de un sistema de telefonía de voz sobre el protocolo de internet el cual es una solución adecuada para la problemática que tiene la empresa Ecuador Overseas Agencies C.A. con la central telefónica IP actual en funcionamiento, siendo un gran impulso para la mejora de la organización. Antes de llevar a cabo la implementación alterna del sistema de voz sobre IP, se realizó un estudio acerca de los recursos que serán usados en el proyecto, como los servidores, teléfonos IP, Softphone, buenas prácticas de seguridad aplicadas a centrales telefónicas IP usando técnicas de hardening que fortalecerán las vulnerabilidades en los servidores para evitar posibles ataques, y software open source que permita mejorar el flujo de las llamadas telefónicas, de esta manera poder demostrar que una solución alternativa con tecnologías open source logra satisfacer las necesidades de la empresa y ahorrar costos para una implementación futura.

Palabras claves: Hardening; virtualización; múltiples servidores VoIP; central telefónica IP; open source. 


\section{Abstract}

The telephone service is an indispensable communication medium in companies that allows to carry out all type of commercial or personal activity. The use of this technology lies in: the quality of service offered at a low cost, availability at all times, accessibility without interruptions or congestion and ease of use without leaving aside security and scalability over time. Due to the constant technological advances and the innovation of opensource technologies and virtualization, it is suggested the implementation of a voice telephony system on the internet protocol, which is a suitable solution for the problems that have the company Ecuador Overseas Agencies C.A. With the current IP telephone exchange in operation, being a great impetus for the improvement of the organization. Before carrying out the alternate implementation of the voice over IP system, a study was made of the resources that will be used in the project, such as servers, IP phones, Softphone, good security practices applied to IP telephone exchanges using techniques Of hardening that will strengthen the vulnerabilities in the servers to avoid possible attacks, and open source software that allows to improve the flow of the telephone calls, in this way to be able to demonstrate that an alternative solution with open source technologies manages to satisfy the needs of the company and to save costs for future implementation.

Key words: Hardening, virtualization, multiple VoIP servers, IP PBX, open source. 


\section{Resumo}

O serviço telefônico é um meio vital de comunicação nas empresas que permite que qualquer tipo de negócio ou atividade pessoal. O uso desta tecnologia encontra-se em: a qualidade do serviço oferecido a um baixo custo, a disponibilidade em todos os momentos, a acessibilidade sem interrupção ou congestionamento e facilidade de uso sem deixar de lado a segurança e escalabilidade ao longo do tempo. Por causa das múltiplas constantes avanços tecnológicos e inovação de tecnologias de código aberto e virtualização, sugere-se a implementação de um sistema de Voice over Internet Protocol, que é uma solução adequada para os problemas que a companhia Equador Agências no exterior CA. com a actual PBX IP em execução, sendo um grande impulso para a melhoria da organização. Antes de efectuar a implementação do sistema alternativo de Voz sobre IP, foi realizado um estudo sobre os recursos a serem utilizados no projeto, como servidores, telefones IP, Softphone, boas práticas de segurança aplicadas a centrais telefônicas IP usando técnicas endurecimento que irá fortalecer vulnerabilidades em servidores para prevenir possíveis ataques e software de código aberto que irão melhorar o fluxo de chamadas telefónicas, portanto, capaz de demonstrar que uma solução alternativa com tecnologias opensource capaz de satisfazer as necessidades do negócio e reduzir os custos para implementação futura.

Palavras-chave: Endurecimento, virtualização, vários servidores VoIP, IP PBX, de código aberto. 


\section{Introducción.}

El servicio de telefonía IP que tenemos actualmente es considerado universal y vital, ya que ha tenido grandes avances con el transcurso del tiempo y gracias a los avances tecnológicos en el área informática es posible él envió de paquetes de voz a través de redes de datos llamados voz sobre IP (VoIP).

Esta tecnología emergente está a nivel mundial ya implementada en diferentes países y consiste en brindar los mismos servicios de telefonía la tradicional pero usando protocolos de red TCP/IP, esta es la razón por la que este tipo de tecnología se ha convertido en un servicio vital para las organizaciones públicas y privadas.

Sin embargo, en el mundo empresarial los costos de las implementaciones suelen tener costos altos que no están al alcance de muchas empresas, y pasan a tener una prioridad baja dependiendo de las necesidades que estas requieran, dado a los costos de adquisición de los equipos físicos y licencias. Una alternativa es la virtualización de estos servidores con los servicios de central telefónica; con la virtualización tenemos las siguientes ventajas como:

Consolidación de Recursos.

Uso y costo menor energía.

Ahorros de espacio.

Rápida incorporación de nuevos recursos para los servidores

La virtualización se asocia con varios conceptos como poder gestionar de mejor manera los recursos, el aumento de protección en las redes, capacidad de aumentar a través del tiempo y fácil administración. Los procesadores en su avance tecnológico han alcanzado un límite donde se aprovecha el $20 \%$ y $30 \%$ del poder total, el principal objetivo es poder tener el uso de múltiples sistemas operativos funcionando al mismo tiempo usando una plataforma común de hardware.

Por lo que las aplicaciones que virtualizan los sistemas operativos dividen las funciones básicas que realiza el sistema como son la multiprogramación y además la abstracción del hardware siendo la técnica de virtualización una alternativa que nos permite alcanzar un objetivo específico: El disminuir los costos totales de operación de las plataformas de servidores. 
Las centrales telefónicas en los últimos años están teniendo un auge en las pequeñas y medianas empresas en relación a la telefonía convencional por todas las ventajas que estas ofrecen, pero ¿Que hace que una empresa cambie su tecnología telefónica?

Cada vez más empresas eligen Internet para sus comunicaciones telefónicas, sobre todo para ahorrarse un dinero en sus facturas. Y es que, los servicios de voz sobre IP (VoIP) permiten a las empresas realizar llamadas a través de la misma red que utilizan para acceder a Internet y al correo electrónico, y con un coste mucho menor que el de redes de voz tradicionales. (Arantxa, 2014)

Pero elegir la tecnología correcta también lleva a cabo un análisis de: ¿Qué realmente necesito de una central VoIP?

Por otro lado, las funcionalidades que requiere una Pyme para su central consisten básicamente en poder recibir y realizar llamadas, tener buzones de voz, un IVR sencillo, colas, capturas de llamadas, transferencias con y sin consulta, música en espera, y poco más. (Varela, 2010)

Mediante esta proyecto de investigación se presenta un esquema de virtualización con un servidor open source que contiene centrales telefónicas las cuales reposan sobre una misma plataforma de hardware, que tendrán políticas de seguridad (Hardening) aplicadas a centrales telefónicas para limitar las vulnerabilidades a las cuales están expuestas; todo esto como una solución alternativa a una central telefónica ya implementada en la empresa Ecuador Overseas Agencies C.A.; demostrando las ventajas de tener un servicio de telefonía VoIP en cuanto a escalabilidad y costos de implementación con tecnología open source y virtualización.

\section{Marco Teórico y Estado del Arte.}

\section{Telefonía IP.}

La telefonía IP está en aumento cada vez más en los últimos años, debido a las bondades y ventajas que ofrecen estos sistemas en comparación a las telefonías convencionales, poco a poco 
están siendo desplazadas, dado que estas no ofrecen movilidad de usuarios, comunicación e interconexión entre departamentos y empresas sin necesidad de pagar valores adicionales. Además, en la actualidad las centrales telefónicas convencionales están siendo desplazadas por las centrales IP por las prestaciones que estas ofrecen, como integración con otras plataformas de software y demás.

En el Ecuador esta tendencia ha ido aumentando en los últimos años en las empresas Pymes. José Estrada, Mayra Calva y Ana Rodríguez en 2016 afirman lo siguiente: Antes de 2009 no se encontraron procesos relacionados con la compra de telefonía IP (sistemas, componentes o servicio). En este año 2016, se ejecutaron 6 procesos de compra y en 2013 llegaron a ser 24. La inversión total realizada en los rubros descritos, de acuerdo a los datos obtenidos, casi alcanza los 28 millones de dólares.

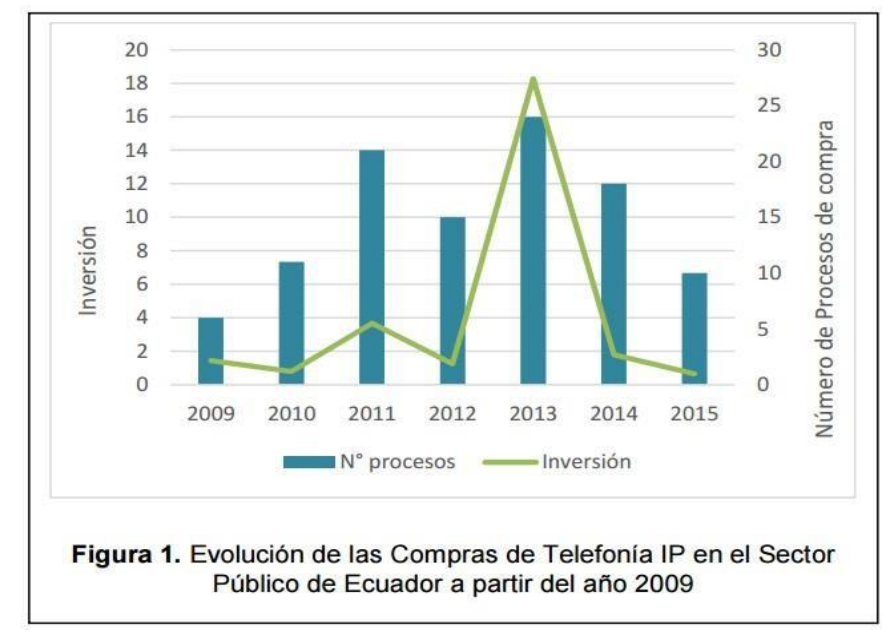

Gráfico 1: Evolución de adquisiciones de Centrales Telefónicas IP desde el 2009 aplicadas al sector público del Ecuador

Fuente: (Estrada, Calva, \& Rodríguez, 2016)

Como se observa en la Figura 1, la inversión en telefonía IP pasó de cero a 16 millones de dólares en 4 años (2009 a 2013) en el sector público, luego de lo cual ésta se ha ido reduciendo paulatinamente. Esta información refleja claramente la tendencia creciente en la utilización de telefonía IP que inicia de manera tardía en comparación con otros países más desarrollados tecnológicamente. Las provincias con mayor cantidad de procesos de compra de telefonía IP son 
Pichincha (56\%), Guayas (14\%), Azuay (10\%) y Tungurahua (6\%) de un total de alrededor de 105 procesos realizados durante los últimos 7 años. (Estrada, Calva, \& Rodríguez, 2016)

\section{Caso de estudio: Empresa Overseas Agencies C.A.}

La empresa inició sus actividades en 1934 bajo el liderazgo y la visión de Juan Schotel Reinberg, ciudadano holandés que emigró a Ecuador para establecerse y desarrollar sus actividades profesionales, la empresa tiene más de 80 años en el mercado de insumos y equipos médicos. Siendo líderes a nivel nacional en instalación, mantenimiento preventivo y correctivo de equipos y provisión de insumos médicos se diferencian por el compromiso y dedicación al mejoramiento de la calidad de salud.

El gerente Juan Martin Schotel, lleva a cabo estrictos controles en los procesos de la empresa para el aseguramiento de calidad, actualmente su principal matriz se encuentra ubicada en la ciudad de Guayaquil, cuenta con un personal mayor a 100 personas con dos sucursales en ecuador, su esquema organizacional comprende una estructura vertical que se segmentan en gerencias y jefaturas a cargo de planificar y controlar las principales actividades del día a día. La misión y visión principal de Ecuador Overseas Agencies C. A. es la calidad en la atención a sus clientes.

La empresa cuenta con una central telefónica Avaya IP adquirida hace ya dos años que fue implementada por un proveedor local, debido a que el proveedor de telefonía IP tiene un código cerrado, solo permite tener una central IP funcionando por licencia, lo que causa que no se puedan realizar pruebas de mejoras en las centrales, y que en cualquier cambio o modificación sea necesario requerir a las personas que implementaron el proyecto, las cuales son ajenas a la empresa. Además, exige tener un servidor físico atado a esta licencia, por lo que no se puede virtualizar la central. Adicional a esto, los servicios por parte del proveedor a cargo del proyecto fueron solo de implementación del software de central telefónica y no se analizó la seguridad.

Estas limitantes permite evidenciar el analizar el caso de la empresa enunciada, se lleva a realizar este proyecto de investigación, donde se ofrece una alternativa a la solución actual, usando tecnologías open source y virtualización que permitirían tener una flexibilidad a la empresa, y adicional darle mecanismos de seguridad efectivos para que no existan ataques o lugares donde puedan entrar agentes maliciosos. 


\section{Formulación del problema.}

¿Puede la virtualización de centrales telefónicas usando múltiples plataformas con técnicas de Hardening ofrecer un mejor servicio de telefonía IP que reduzca los costos de implementación y mejore la comunicación en la empresa Ecuador Overseas Agencies C.A.?

\section{Virtualización.}

Este proyecto trata sobre la virtualización, pero ¿Que es la virtualización?, muchas personas y empresas solo tienen conocimientos generales, pero cuando se quiere profundizar acerca de las ventajas y beneficios que se pueden obtener generan dudas que no saben responder.

Este es el principal uso que se le está dando a la virtualización en las empresas: Se busca un servidor potente a nivel de hardware, que nos permita alojar y ejecutar varios servidores virtuales dedicados a distintos propósitos. Además del ahorro económico que supone sólo en consumo eléctrico la principal ventaja de la virtualización viene asociada con la administración de los equipos. Por lo tanto, la virtualización nos ayuda a mantener la continuidad de negocio que para muchas empresas que no se pueden permitir paradas en sus sistemas, o que dichas paradas tienen un coste elevado rentabilizan los recursos invertidos en la virtualización. ([BBVA], 2012)

Analizando el tema de virtualización, también debemos hacer referencia que un servidor óptimo no solo es aquel que lleva sus funciones de manera correcta, sino aquel que tiene las suficientes seguridades para evitar la fuga de información. La seguridad en servidores es la clave para tener servidores seguros. Todos los elementos que participan en un sistema de información pueden verse afectados por fallos de seguridad (...). El hardware y otros elementos físicos se pueden volver a comprar, el software puede ser reinstalado, pero la información dañada o robada no siempre es recuperable. (Purificación, 2010)

El motivo de llevar a cabo una virtualización es poder dar uso de varias sistemas operativos sobre uno que ya existe, sin que las funcionalidades o características de este se vean afectados con un arranque independiente gracias al software de virtualización llamado Virtual Machine Monitor o VMM que crea una abstracción entre el software del Sistema Operativo y el equipo físico. 


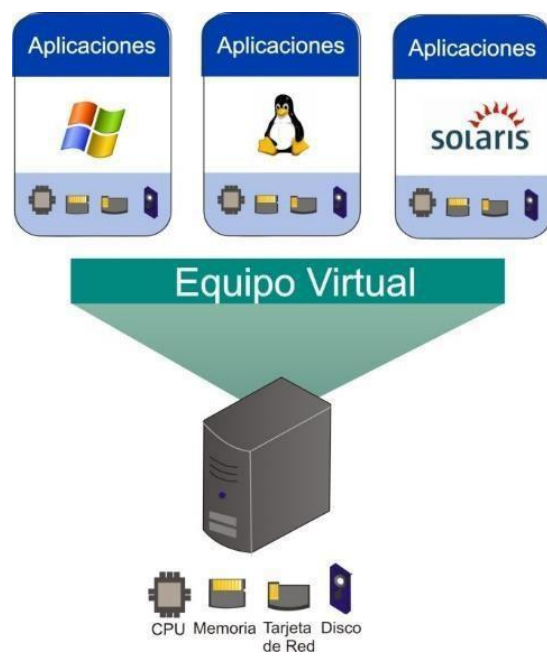

Gráfico 2: Virtualización

Fuente: http://sitproyectos.com/productos-y-servicios/servicios-y- solucionesit/virtualizacion

\section{Hardening.}

Es el proceso que lleva a cabo técnicas y medidas de seguridad para endurecer un sistema con el objetivo de cerrar agujeros de seguridad, eliminar servicios innecesarios y fortalecer el control de acceso para evitar en lo posible ataques que dejen el sistema funcionando de una manera incorrecta o indisponible.

Haciéndole la vida difícil al atacante. Ese es el concepto que está detrás del Hardening de sistemas operativos. Hardening es una acción compuesta por un conjunto de actividades que son llevadas a cabo por el administrador de un sistema operativo para reforzar al máximo posible la seguridad de su equipo. Su propósito, entorpecer la labor del atacante y ganar tiempo para poder minimizar las consecuencias de un inminente incidente de seguridad e incluso, en algunos casos, evitar que éste se concrete en su totalidad. (Montenegro, 2012)

Entre los objetivos del Hardening son: Minimizar las vulnerabilidades del sistema, disminuir el impacto de un incidente de seguridad, mejorar la administración del sistema al identificar de una manera más rápida la causa de un incidente al poder descartar las causas que ya se tomaron acciones con el proceso de Hardening. 


\section{Materiales y métodos.}

\section{Modalidad de la Investigación}

Para el correcto desenvolvimiento del proyecto se ha utilizado la modalidad de investigación aplicada, porque se basa en la utilización de los conocimientos en la práctica para resolver un problema y por lo general es en beneficio de la sociedad, como (Leyton, 2012) afirma: "La Investigación aplicada busca el conocer, para hacer, para actuar, para construir y para modificar”.

\section{Tipo de investigación}

En el proyecto de titulación se utiliza el tipo de investigación explicativa, la cual su objetivo es explicar la relación que tienen una variable con otra es decir como las variables dependientes se pueden modificar si cambia el estado de la variable independiente, se basa en identificar las causas del problema para determinar las consecuencias y como se relacionan entre sí. Según nos indica (Marulanda, 2011) "Investigación explicativa es la explicación que trata de descubrir, establecer y explicar las relaciones causalmente funcionales que existen entre las variables estudiadas, y sirve para explicar cómo, cuándo, dónde y por qué ocurre un fenómeno social”.

\section{Población y muestra}

\section{Población}

La población está definida por los usuarios finales, personal técnico y directivo de la empresa Ecuador Overseas Agencies C.A, según información obtenida es de 76 personas quienes emplearán el proyecto a quienes se realizan entrevistas y encuestas.

\section{Software y hardware}

- Asterick

- Elastix

- Teléfono Avaya $9608 \mathrm{G}$

- Softphone Avaya Flare 


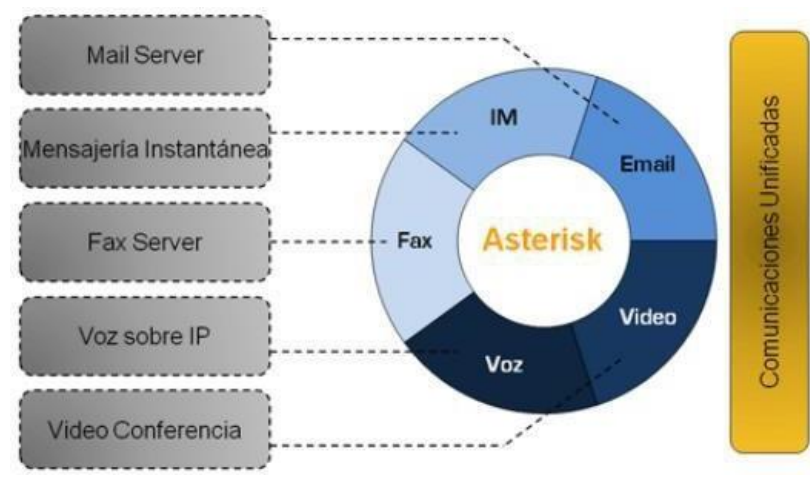

Gráfico 3: Estructura de Elastix

Fuente: Curso de Entrenamiento Elastix (Anaya, 2013)

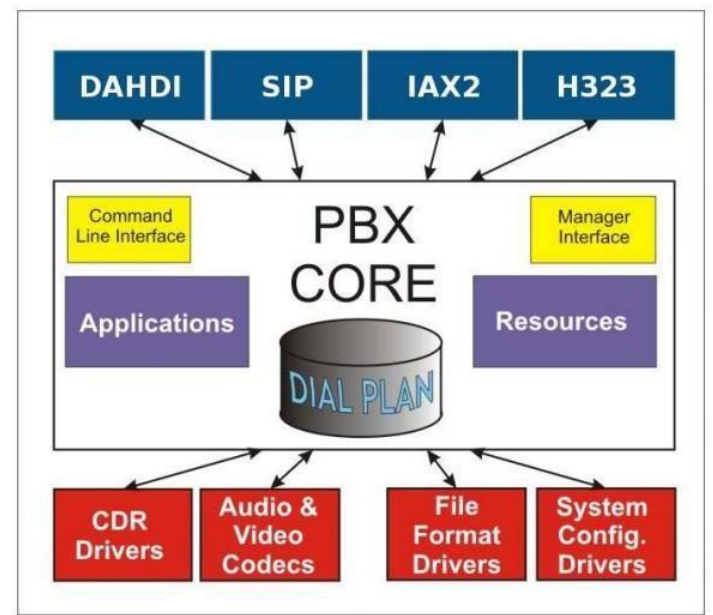

Gráfico 4: Estructura de Asterick

Fuente: Fundamentos de Telefonía IP e Introducción a Asterisk/Elastix (Anaya, 2013)

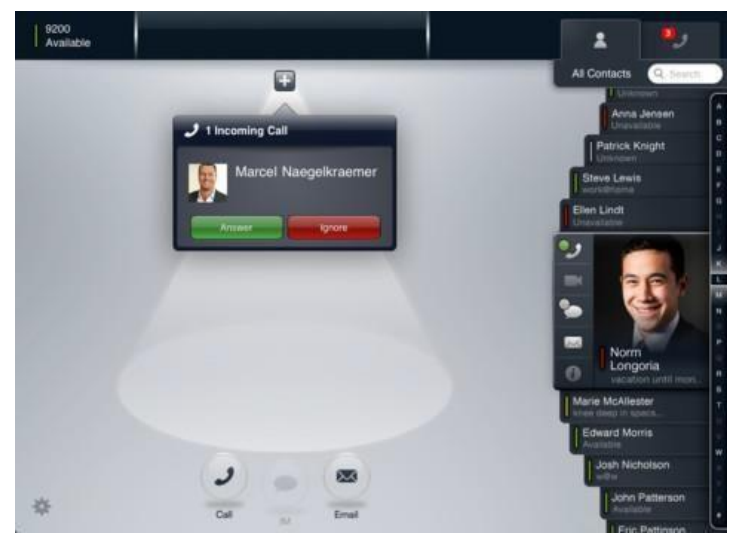

Gráfico 5: Softphone Avaya Flare

Fuente: https://downloads.avaya.com/css/P8/documents/100175103 
Eleanor A. Varela-Tapia; Diana G. Alban-Loor; Iván L. Acosta-Guzmán; Jenniffer M. Yagual-Lozano

\section{Resultados y Discusión.}

Solución propuesta tecnológica del proyecto

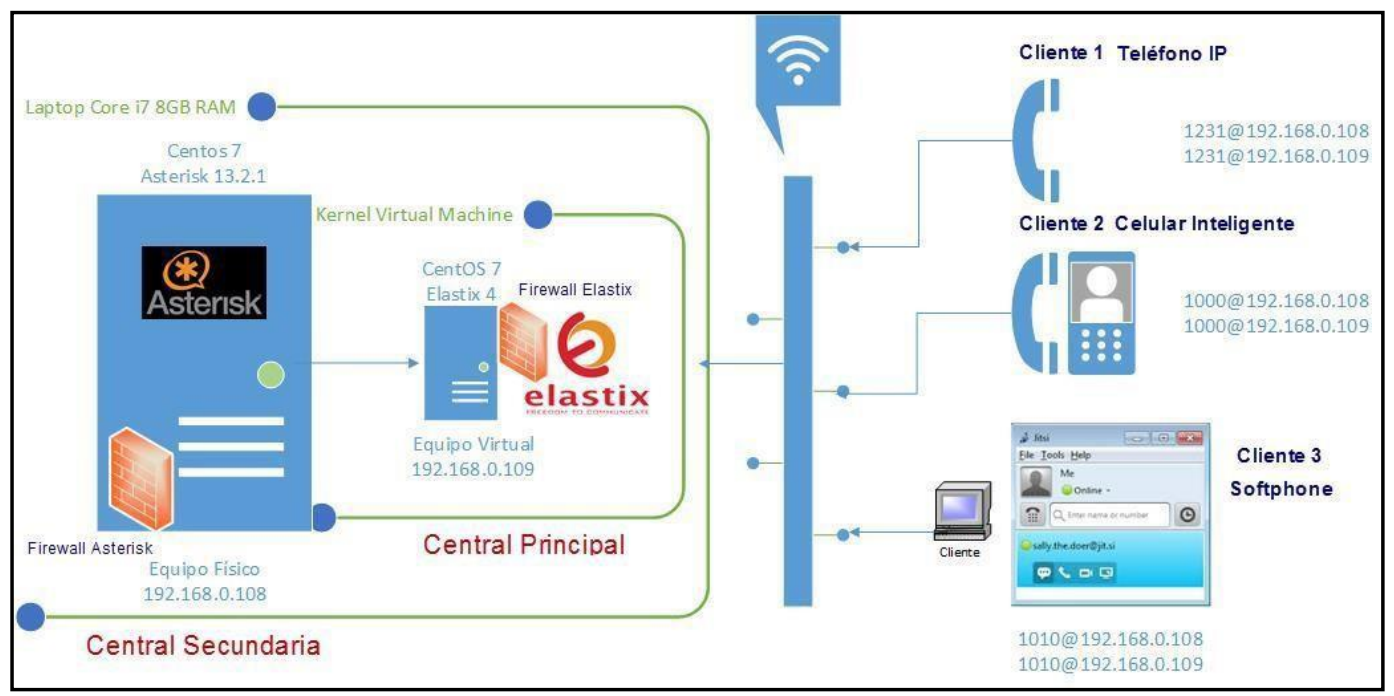

Gráfico 6: Diagrama de la Arquitectura

Fuente: Proyecto de investigación

Aplicación informática

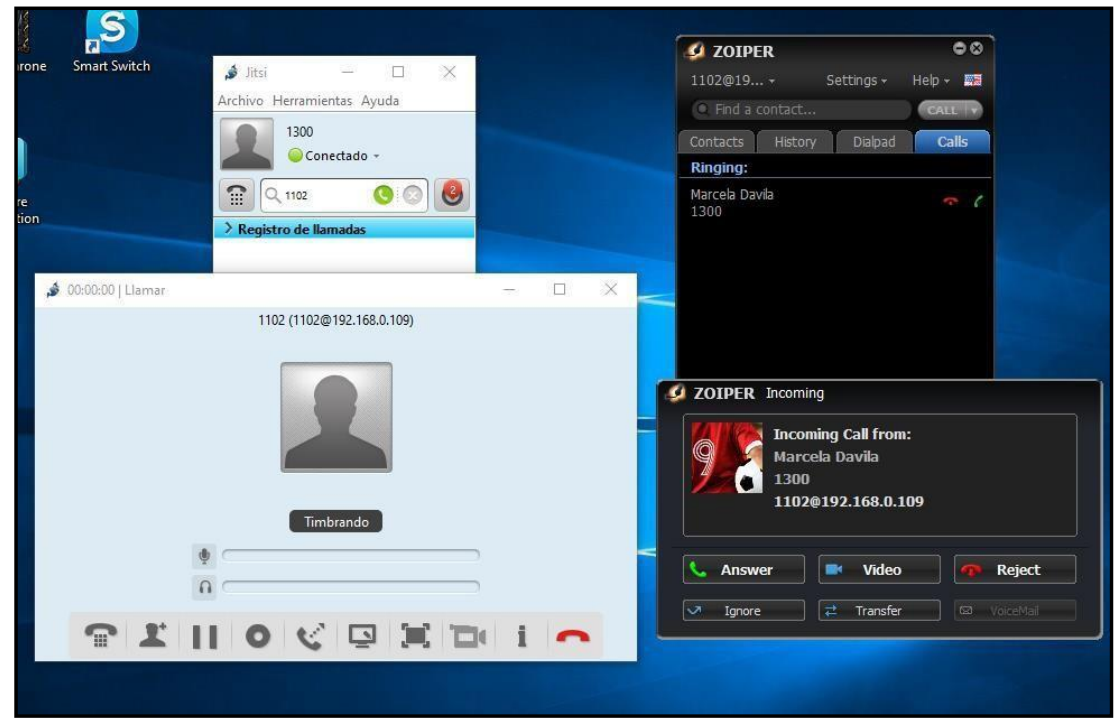

Gráfico 7: Visualización de llamada en la PC Fuente: Proyecto de investigación 
De las encuestas

El motivo de haber elegido el cuestionario como instrumento fue para obtener datos que serán tabulados y llegar a un análisis específico en el cual se establecerán los siguientes mecanismos:

- $\quad$ Por cada pregunta de la encuesta se realizara la tabulación de la información obtenida.

- Se realiza el diseño de cuadros estadísticos para mejor visualización de los datos.

- Interpretación y análisis detallado de cada resultado obtenido en las preguntas planteadas.

Resultado del análisis generado por los cuestionarios Preguntas realizadas a los directivos de Ecuador Overseas Agencies C.A.

1.- ¿Cómo evaluaría de manera general el rendimiento y prestaciones de la central telefónica implementada en la empresa?

\begin{tabular}{|c|c|c|}
\hline Opciones & Cantidad & Porcentaje \\
\hline Muy Bueno & 0 & $0.00 \%$ \\
\hline Bueno & 0 & $0.00 \%$ \\
\hline Neutro & 1 & $100.00 \%$ \\
\hline Malo & 0 & $0.00 \%$ \\
\hline Muy Malo & 0 & $0.00 \%$ \\
\hline Total & $\mathbf{1}$ & $\mathbf{1 0 0 . 0 0 \%}$ \\
\hline
\end{tabular}

Resultados Pregunta 1 Directivos Ecuador Overseas

Cuadro N. 1

Fuente: Investigación del tema planteado

Análisis: De acuerdo a la respuesta dada, podemos determinar que el nivel de satisfacción es neutral, esto denota que no está convencido por la solución implementada, debido que no cumple al $100 \%$ las expectativas esperadas, y a la vez nos motiva a continuar con el proyecto como una solución alternativa. 
Eleanor A. Varela-Tapia; Diana G. Alban-Loor; Iván L. Acosta-Guzmán; Jenniffer M. Yagual-Lozano

2.- ¿En qué porcentaje la central telefónica implementada ha cumplido con las necesidades de lo que se esperaba vs lo implementado anterior?

\begin{tabular}{|c|c|c|}
\hline Opciones & Cantidad & Porcentaje \\
\hline Igual a $100 \%$ & 0 & $0.00 \%$ \\
\hline Entre 75 y $99 \%$ & 0 & $0.00 \%$ \\
\hline Entre 50 y $74 \%$ & 1 & $100.00 \%$ \\
\hline Entre 25 y $49 \%$ & 0 & $0.00 \%$ \\
\hline Menor a $25 \%$ & 0 & $0.00 \%$ \\
\hline Total & $\mathbf{1}$ & $\mathbf{1 0 0 . 0 0} \%$ \\
\hline
\end{tabular}

Resultados Pregunta 2 Directivos Ecuador Overseas

\section{Cuadro N. 2}

Fuente: Investigación del tema planteado

Análisis: De acuerdo a la respuesta dada se puede denotar el porcentaje de cumplimiento respecto a las necesidades requeridas esta entre el $74 \%$ y $50 \%$, esto nos da una idea de que aunque no funciona en su totalidad la central telefónica IP, solo usan las funciones básicas, esto es un indicador que necesita llegar al $100 \%$ con la solución alternativa planteada.

3.- ¿En qué porcentaje cree Ud. que ha mejorado el servicio de llamadas en la empresa con la central telefónica actual?

\begin{tabular}{|c|c|c|}
\hline Opciones & Cantidad & Porcentaje \\
\hline Igual a $100 \%$ & 0 & $0.00 \%$ \\
\hline Entre 75 y $99 \%$ & 0 & $0.00 \%$ \\
\hline Entre 50 y $74 \%$ & 1 & $100.00 \%$ \\
\hline Entre 25 y $49 \%$ & 0 & $0.00 \%$ \\
\hline Menor a $25 \%$ & 0 & $0.00 \%$ \\
\hline Total & $\mathbf{1}$ & $\mathbf{1 0 0 . 0 0 \%}$ \\
\hline
\end{tabular}

Resultados Pregunta 3 Directivos Ecuador Overseas

Cuadro N. 3

Fuente: Investigación del tema planteado

Análisis: Desde la fecha de implementación de la central telefónica hasta la actualidad, no se han visto mejoras en su totalidad, ya que existen quejas por parte de los usuarios y los clientes de la empresa también. 
4.- Ha tenido alguna queja por parte de los clientes respecto al servicio de telefonía recién implementada

\begin{tabular}{|c|c|c|}
\hline Opciones & Cantidad & Porcentaje \\
\hline SI & 1 & $100.00 \%$ \\
\hline NO & 0 & $0.00 \%$ \\
\hline Total & $\mathbf{1}$ & $\mathbf{1 0 0 . 0 0 \%}$ \\
\hline
\end{tabular}

Resultados Pregunta 4 Directivos Ecuador Overseas

Cuadro N. 4

Fuente: Investigación del tema planteado

Análisis: De acuerdo a esta respuesta, la gerencia en sus reuniones con clientes por temas comerciales si han tenido quejas desde el inicio de la nueva central y es un punto en contra para la empresa, porque los clientes sienten el malestar de comunicarse para obtener un servicio.

5.- ¿Cómo consideraría Ud. el gasto que genero la central telefónica implementada en relación a los gastos generales de la empresa?

\begin{tabular}{|c|c|c|}
\hline Opciones & Cantidad & Porcentaje \\
\hline Muy Alto & 1 & $100.00 \%$ \\
\hline Alto & 0 & $0.00 \%$ \\
\hline Neutro & 0 & $0.00 \%$ \\
\hline Bajo & 0 & $0.00 \%$ \\
\hline Muy Bajo & 0 & $0.00 \%$ \\
\hline Total & $\mathbf{1}$ & $\mathbf{1 0 0 . 0 0} \%$ \\
\hline
\end{tabular}

Resultados Pregunta 5 Directivos Ecuador Overseas

Cuadro N. 5

Fuente: Investigación del tema planteado

Análisis: Como podemos observar en el cuadro, el costo de la inversión generada por la empresa por una solución que satisficiera sus necesidades fue muy alto vs la realidad que está pasando, esto se considera un gasto considerable, ya que no genera una rentabilidad o retorno de inversión. 
6.- ¿Tiene conocimiento del uso de centrales telefónicas con tecnología open source, que no tienen un costo por licencia y ofrecen servicios iguales a los proveedores conocidos?

\begin{tabular}{|c|c|c|}
\hline Opciones & Cantidad & Porcentaje \\
\hline SI & 1 & $100.00 \%$ \\
\hline NO & 0 & $0.00 \%$ \\
\hline Total & $\mathbf{1}$ & $\mathbf{1 0 0 . 0 0 \%}$ \\
\hline
\end{tabular}

\section{Resultados Pregunta 6 Directivos Ecuador Overseas \\ Cuadro N. 6}

Fuente: Investigación del tema planteado

Análisis: En los procesos de adquisición de la central actual, hubo propuestas que también incluyeron soluciones open source, y el departamento de TI puso a su conocimiento cuales son ventajas y desventajas de cada uno de las características que tienen.

7.- Si le ofrecieran una solución de central telefónica que pueda mejorar el servicio de manera sencilla, y además que no genere los costos por licenciamiento, ¿estaría de acuerdo en implementarla?

\begin{tabular}{|c|c|c|}
\hline Opciones & Cantidad & Porcentaje \\
\hline SI & 1 & $100.00 \%$ \\
\hline NO & 0 & $0.00 \%$ \\
\hline Total & $\mathbf{1}$ & $\mathbf{1 0 0 . 0 0 \%}$ \\
\hline
\end{tabular}

\section{Resultados Pregunta 7 Directivos Ecuador Overseas}

Cuadro N. 7

Fuente: Investigación del tema planteado

Análisis: En esta respuesta vemos en comparación a la pregunta anterior, donde los directivos tienen dudas acerca de las plataformas open source, están dispuestos a implementar una solución alternativa que estamos proponiendo, lo cual nos da esa apertura a continuar con nuestro proyecto. 


\section{Encuesta al Departamento de Sistemas}

1.- En qué porcentaje del tiempo de trabajo recibe quejas de la central telefónica actualmente implementada.

\begin{tabular}{|c|c|c|}
\hline Opciones & Cantidad & Porcentaje \\
\hline Igual a $100 \%$ & 0 & $0.00 \%$ \\
\hline Entre 75 y $99 \%$ & 1 & $50.00 \%$ \\
\hline Entre 50 y $74 \%$ & 1 & $50.00 \%$ \\
\hline Entre 25 y 49\% & 0 & $0.00 \%$ \\
\hline Menor a 25\% & 0 & $0.00 \%$ \\
\hline Total & $\mathbf{2}$ & $\mathbf{1 0 0 . 0 0 \%}$ \\
\hline
\end{tabular}

Resultados Pregunta 1 Dpto. Sistemas

Cuadro N. 8

Fuente: Investigación del tema planteado

Análisis: Podemos constatar que más del 50\% del tiempo de trabajo se reciben quejas por la central telefónica actual, lo cual genera pérdida de tiempo en otras actividades que se podrían estar realizando.

2.- ¿Cómo evaluaría usted el uso y rendimiento de la central telefónica actualmente implementada en la empresa?

\begin{tabular}{|c|c|c|}
\hline Opciones & Cantidad & Porcentaje \\
\hline Muy Bueno & 0 & $0.00 \%$ \\
\hline Bueno & 1 & $50.00 \%$ \\
\hline Neutro & 1 & $50.00 \%$ \\
\hline Malo & 0 & $0.00 \%$ \\
\hline Muy Malo & 0 & $0.00 \%$ \\
\hline Total & $\mathbf{2}$ & $\mathbf{1 0 0 . 0 0 \%}$ \\
\hline
\end{tabular}

Resultados Pregunta 2 Dpto. Sistemas

Cuadro N. 9

Fuente: Investigación del tema planteado

Análisis: Podemos observar que el departamento de sistemas no tiene un buen concepto de la central debido a los constantes cambios e inconvenientes que ha generado desde la implementación. 
Eleanor A. Varela-Tapia; Diana G. Alban-Loor; Iván L. Acosta-Guzmán; Jenniffer M. Yagual-Lozano

3.- ¿Cuáles del siguiente software para centrales telefónicas son conocidos por usted?

\begin{tabular}{|c|c|c|}
\hline Opciones & Cantidad & Porcentaje \\
\hline Asterisk & 1 & $50.00 \%$ \\
\hline Avaya & 1 & $50.00 \%$ \\
\hline 3Com & 0 & $0.00 \%$ \\
\hline Elastix & 2 & $100.00 \%$ \\
\hline Cisco & 0 & $0.00 \%$ \\
\hline Panasonic & 2 & $100.00 \%$ \\
\hline $3 C X$ & 0 & $0.00 \%$ \\
\hline Polycom & 0 & $0.00 \%$ \\
\hline LyncServer & 1 & $50.00 \%$ \\
\hline Otros & 0 & $0.00 \%$ \\
\hline Total & 7 & \\
\hline
\end{tabular}

Fuente: Investigación del tema planteado

Análisis: La figura nos muestra que Elastix y Panasonic son las tecnologías de centrales IP más conocidas por el dpto. De sistemas, al ser Elastix una de las soluciones a implementar, será de mucho beneficio en la curva de aprendizaje.

4.- La empresa actual maneja algún sistema basado en uso de licencias open source

\begin{tabular}{|c|c|c|}
\hline Opciones & Cantidad & Porcentaje \\
\hline SI & 0 & $0.00 \%$ \\
\hline NO & 1 & $100.00 \%$ \\
\hline Total & $\mathbf{1}$ & $\mathbf{1 0 0 . 0 0 \%}$ \\
\hline
\end{tabular}

$$
\begin{gathered}
\text { Resultados Pregunta } 4 \text { Dpto. Sistemas } \\
\text { Cuadro N. } 11
\end{gathered}
$$

Fuente: Investigación del tema planteado

Análisis: De acuerdo a la respuesta, la empresa no posee ningún software de ningún tipo que sea open source, en caso que la solución se implemente será la primera en la empresa; que será algo innovador que ayudara a mejorar su gestión de telefonía IP. 
5.- En qué porcentaje considera usted el nivel de importancia que tienen la seguridad de los servidores de central telefónica

\begin{tabular}{|c|c|c|}
\hline Opciones & Cantidad & Porcentaje \\
\hline Igual a $100 \%$ & 1 & $50.00 \%$ \\
\hline Entre 75 y $99 \%$ & 1 & $50.00 \%$ \\
\hline Entre 50 y $74 \%$ & 0 & $0.00 \%$ \\
\hline Entre 25 y $49 \%$ & 0 & $0.00 \%$ \\
\hline Menor a $25 \%$ & 0 & $0.00 \%$ \\
\hline Total & $\mathbf{2}$ & $\mathbf{1 0 0 . 0 0 \%}$ \\
\hline
\end{tabular}

Resultados Pregunta 5 Dpto. Sistemas

Cuadro N. 12

Fuente: Investigación del tema planteado

Análisis: Para el dpto. De Sistemas de la compañía Ecuador Overseas la importancia de tener sistemas con una buena seguridad pasa el $75 \%$, ya que los servicios y sistemas deben estar debidamente adecuados para evitar ataques. Nuestro proyecto cuenta con las seguridades para que reducir las vulnerabilidades.

6.- Si le ofrecieran una solución de central telefónica virtualizada usando mecanismo de seguridad y hardening, que sea de fácil uso e implementación, ¿cree usted que mejoraría el servicio de telefonía actual?

\begin{tabular}{|c|c|c|}
\hline Opciones & Cantidad & Porcentaje \\
\hline $\mathrm{SI}$ & 1 & $100.00 \%$ \\
\hline $\mathrm{NO}$ & 0 & $0.00 \%$ \\
\hline Total & $\mathbf{1}$ & $\mathbf{1 0 0 . 0 0} \%$ \\
\hline
\end{tabular}

Resultados Pregunta 6 Dpto. Sistemas

Cuadro N. 13

Fuente: Investigación del tema planteado

Análisis: Como podemos observar, el dpto. De sistemas cree al 100\% que si se ofrece una solución alternativa de fácil uso puede mejorar el servicio de telefonía y además reducir la cantidad de incidencias que se registran durante largos periodos por la central telefónica actual. 
Eleanor A. Varela-Tapia; Diana G. Alban-Loor; Iván L. Acosta-Guzmán; Jenniffer M. Yagual-Lozano

\section{Encuesta a los empleados de Ecuador Overseas}

1.- De acuerdo a la siguiente escala, ¿cómo calificaría el servicio de telefonía en la empresa?

\begin{tabular}{|c|c|c|}
\hline Opciones & Cantidad & Porcentaje \\
\hline Excelente & 7 & $9.59 \%$ \\
\hline Bueno & 4 & $5.48 \%$ \\
\hline Neutro & 14 & $19.18 \%$ \\
\hline Malo & 35 & $47.95 \%$ \\
\hline Muy Malo & 13 & $17.81 \%$ \\
\hline Total & $\mathbf{7 3}$ & $\mathbf{1 0 0 . 0 0 \%}$ \\
\hline
\end{tabular}

Resultados Pregunta 1 Empleados

Cuadro N. 14

Fuente: Investigación del tema planteado

Análisis: Como podemos ver el cuadro, casi más del 60\% de los empleados que laboran en la empresa considera que el servicio tiende a ser muy malo con el pasar del tiempo. Lo cual genera insatisfacción para los que lo usan.

2.- ¿Cree usted que se debe mejorar la calidad del servicio de telefonía?

\begin{tabular}{|c|c|c|}
\hline Opciones & Cantidad & Porcentaje \\
\hline SI & 63 & $86.30 \%$ \\
\hline NO & 10 & $13.70 \%$ \\
\hline Total & $\mathbf{7 3}$ & $\mathbf{1 0 0 . 0 0 \%}$ \\
\hline
\end{tabular}

Resultados Pregunta 2 Empleados

Cuadro N. 15

Fuente: Investigación del tema planteado

Análisis: Tal como los empleados consideran que el servicio de la telefonía recién implementado es de muy mala calidad, además el 86\% considera que debe mejorar de alguna manera, para evitar futuros inconvenientes al momento de comunicarse. 
3.- ¿Que tan fácil considera usted el uso de la central telefónica actual?

\begin{tabular}{|c|c|c|}
\hline Opciones & Cantidad & Porcentaje \\
\hline Muy Fácil & 9 & $12.33 \%$ \\
\hline Fácil & 6 & $8.22 \%$ \\
\hline Neutro & 11 & $15.07 \%$ \\
\hline Difícil & 32 & $43.84 \%$ \\
\hline Muy Difícil & 15 & $20.55 \%$ \\
\hline Total & $\mathbf{7 3}$ & $\mathbf{1 0 0 . 0 0 \%}$ \\
\hline
\end{tabular}

Resultados Pregunta 3 Empleados

Cuadro N. 16

Fuente: Investigación del tema planteado

Análisis: El cuadro nos indica que el 47\% de los empleados considera que el manejo de los teléfonos fijos como de los Softphones tiene un nivel de complejidad muy alto, lo cual retrasa su trabajo al momento de las llamadas cuando se requiere hacer algo más avanzando.

4.- Si se quedara sin servicio de telefonía por un tiempo alto, ¿cuál es el impacto que tendría respecto a sus labores diarias?

\begin{tabular}{|c|c|c|}
\hline Opciones & Cantidad & Porcentaje \\
\hline Alto & 32 & $43.84 \%$ \\
\hline Poco Alto & 20 & $27.40 \%$ \\
\hline Neutro & 8 & $10.96 \%$ \\
\hline Poco Bajo & 4 & $5.48 \%$ \\
\hline Bajo & 9 & $12.33 \%$ \\
\hline Total & $\mathbf{7 3}$ & $\mathbf{1 0 0 . 0 0 \%}$ \\
\hline
\end{tabular}

Resultados Pregunta 4 Empleados

Cuadro N. 17

Fuente: Investigación del tema planteado

Análisis: El 52\% de los empleados considera que quedarse por un tiempo alto sin telefonía, genera una pérdida de productividad ya que se pueden perder negocios o arreglos comerciales con clientes; lo cual impide que se pueda comunicar de manera efectiva con las extensiones internas y números de clientes y proveedores 
5.- ¿Que tan de acuerdo estaría Ud. en que se implemente una solución alternativa para la central telefónica que mejore la calidad y el flujo de las llamadas?

\begin{tabular}{|c|c|c|}
\hline Opciones & Cantidad & Porcentaje \\
\hline Total Acuerdo & 35 & $47.95 \%$ \\
\hline Acuerdo & 21 & $28.77 \%$ \\
\hline Neutral & 12 & $16.44 \%$ \\
\hline Desacuerdo & 3 & $4.11 \%$ \\
\hline Total Desacuerdo & 2 & $2.74 \%$ \\
\hline Total & $\mathbf{7 3}$ & $\mathbf{1 0 0 . 0 0 \%}$ \\
\hline
\end{tabular}

Resultados Pregunta 5 Empleados

Cuadro N. 18

Fuente: Investigación del tema planteado

Análisis: Tal como vemos en el gráfico, el 56\% de los empleados de la empresa Ecuador Overseas requieren una solución inmediata para el problema de la central telefónica, algunos se quejan que con la central análoga llevaban su trabajo de mejor manera, con la solución que planteamos lograremos satisfacer las necesidades de la empresa que actualmente mantiene.

\section{Justificación de la Propuesta.}

De acuerdo a la encuesta planteada a la empresa Ecuador Overseas Agencies C.A., al gerente general, departamento técnico y empleados que laboran en la compañía podemos determinar que existe de manera general existe una desconformidad hacia la central telefónica, más acentuada en la parte operativa que corresponden a los empleados que en sus actividades laboran haciendo llamadas ya sean a proveedores, clientes, cobros etc. Adicional el impacto que genera la central telefónica IP al no estar en operaciones es grande, porque reduce el trabajo que realiza, el dpto. De IT de la empresa por otro lado indica que los incidentes que se registran por la central actual se ha incrementado, haciendo que parte de su tiempo se dediquen a solucionar los problemas; un punto que se debe denotar, es que tanto gerencia como los empleados y el departamento de IT están de acuerdo en que se busque una solución alternativa que satisfaga sus necesidades de tener una herramienta que genere productividad y elimine desperdicios de tiempo por esperas y trabas que se puede suscitar. 


\section{Conclusiones.}

Una vez realizado el proyecto con la empresa Ecuador Overseas Agencies C.A se ha podido obtener mucha información acerca de la central telefónica IP que actualmente está implementada y como la nueva solución propuesta llega a cubrir esos espacios que la herramienta actual tiene como falencias.

Se han analizado para el proyecto muchas herramientas de virtualización para plataformas open source basadas en Linux/Unix, algunas de estas con funcionalidades y características que difieren de la otra, pero al momento elegir va a depender de las necesidades para la cual se la requiera, porque un software de virtualización puede tener atributos que sean buenos pero no los que buscamos, para nuestro proyecto KVM cumplió con las expectativas planteadas que fue ofrecer un sistema de virtualización con los recursos suficientes para soportar Elastix 4 y mantener el óptimo rendimiento del mismo.

De acuerdo a lo investigado, la virtualización sigue estando en el primer puesto como el método efectivo para dividir recursos computacionales, ya que a partir de una plataforma de hardware podemos dividir varios sistemas independientes que funcionan como si fueran un equipo físico externo además que poseen interconectividad y permiten tener una mejor control, respaldo y redundancia de equipos virtuales, movilidad y facilidad de uso, esto hace que la gestión del personal de TI dedique más tiempo a controles y seguridades que a múltiples configuraciones.

Otro de los puntos evaluados fue la seguridad que se aplican a los servidores, también conocido como Hardening. Los controles que se pueden aplicar a uno o varios servidores de acuerdo al estudio dependerán de cuan seguro queremos que sea el equipo de la mano con las políticas de la empresa a la cual estamos evaluando y sugiriendo. Para el caso de Ecuador Overseas Agencies, se sugiere políticas de seguridad establecidas para servidores, mediante un conjunto de criterios a utilizar y el personal de sistemas definió cuales iban acordes las necesidades requeridas, posterior estas reglas fueron aplicadas a las centrales telefónicas IP del proyecto, las cuales abarcaron la mayor cantidad de defensas para evitar vulnerabilidades por agentes externos, sin interferir en los accesos con los demás equipos de cómputo. 
En las pruebas que se realizaron para validar las fortalezas del Hardening aplicado en los servidores, se dieron resultados favorables en las actividades realizadas, además se encontraron oportunidades de mejora donde se pueden seguir explotando más las seguridades de los equipos de cómputo que el departamento de sistemas tiene a su cargo.

La implementación es una parte fundamental de todo proyecto, para el tema desarrollado se preparó un demo de la solución alternativa usando equipos propios para el uso y configuración de los servidores. Se realizaron las configuraciones de cada central IP independiente las que fueron analizadas con el departamento de sistemas de la empresa. La interacción que mantiene durante un proyecto de implementación con el área de sistemas de la empresa aporta muchos conocimientos además que se pudieron profundizar temas de que mejoras se podían obtener con la solución alternativa que estábamos ofreciendo.

La presentación oficial del proyecto a la empresa Ecuador Overseas se realizó de manera satisfactoria, ya que se cumplieron los tiempos y el objetivo buscado, además que un grupo de usuarios pudieron probar el funcionamiento de la central IP alternativa y el departamento de sistemas tuvo una mejor apreciación acerca de estas dos centrales IP como son Elastix y Asterisk que en su debido momento por falta de conocimiento y profundizar un poco más desconocían de las ventajas que ofrecen, además se tomaron nota de cambios o sugerencias que pueden ser aplicados a las centrales para mejorar su funcionamiento.

De manera general, el desarrollo de proyectos aplicados a empresas como Ecuador Overseas Agencies brinda un amplio conocimiento del funcionamiento de las compañías pymes, su desenvolvimiento en el mundo de los negocios y que tan importante es tener una central telefónica IP que esté disponible en todo momento para el correcto desenvolvimiento de las actividades diarias para ofrecer un servicio de calidad a los clientes internos y externos.

\section{Agradecimiento}

Se agradece a la empresa Ecuador Overseas Agencies C.A., por brindar las facilidades necesarias para realizar el estudio del proyecto de investigación, pudiendo encontrar la solución que permite optimizar el funcionamiento de los servidores mediante el Hardening y la Virtualización para el manejo de múltiples servidores de VoIP sobre una misma plataforma. 


\section{Bibliografía.}

[BBVA], B. B. (25 de abril de 2012). Qué es la virtualización y qué ventajas aporta a las empresas. Obtenido de Qué es la virtualización y qué ventajas aporta a las empresas: http://www.bbvacontuempresa.es/a/que- es-la-virtualización-y-que-ventajas-aporta-a-las-empresas

Anaya, N. (2013). Curso de Entrenamiento Elastix. Obtenido de Manual Teórico Curso Entrenamiento Elastix: http://elastixtech.com/wpcontent/uploads/2013/01/MANUAL-TEORICO-CURSOENTRENAMIENTO-ELASTIX-2013.pdf

Arantxa, A. (2014). Las mejores aplicaciones VoIP para empresas. Obtenido de Las mejores aplicaciones VoIP para empresas: http://www.muypymes.com/2014/09/09/aplicaciones-voip

Avaya. (2013). Fundamentos de Telefonía IP e Introducción a Asterisk/Elastix. Uso de Avaya Flare®. Avaya.

Estrada, J., Calva, M., \& Rodríguez, A. (junio de 2016). Seguridad de la Telefonía IP en Ecuador: Análisis en Internet. Obtenido de Seguridad de la Telefonía IP en Ecuador: Análisis en Internet: ingenieria.ute.edu.ec/enfoqueute/index.php/revista/article/download/93/98

Leyton, A. (19 de mayo de 2012). PORQUE INVESTIGACION ES TODO Y TODO ES INVESTIGACION. Obtenido de Clases y tipos de Investigación Científica: https://investigacionestodo.wordpress.com/2012/05/19/clases- y-tipos-de-investigacion-cientifica/

Linux, M. (2008). Decreto 1014 Software Libre Ecuador. Obtenido de https://drwn.wordpress.com/2008/04/11/decreto-1014-software-libre-en- ecuador/

MARULANDA, N. L. (15 de MARZO de 2011). UNIVERSIDAD AMERICA. Obtenido de CLASES Y TIPOS DE INVESTIGACION Y S SUS CARACTERISTICAS: http://www.academia.edu/5075869/CLASES_Y_TIPOS_DE_INVESTIGACION_Y_SUS_CARAC TERISTICAS

Montenegro, L. (3 de abril de 2012). Proceso de Hardening. Obtenido de https://seguinfo.wordpress.com/2012/04/03/proceso-de-hardening/

Purificación, A. (2010). Seguridad informática. Editex.

http://sitproyectos.com/productos-y-servicios/servicios-y- soluciones-it/virtualizacion

https://downloads.avaya.com/css/P8/documents/100175103

Varela, M. (2010). ¿Cuál es la PBX IP más adecuada para PYMES? Obtenido de ¿Cuál es la PBX IP más adecuada para PYMES?: https://www.sinologic.net/blog/2010-03/\%C2\%BFcual-es-la-pbx-ip-masadecuada-parapymes.html 